\title{
Dedifferentiated Dermatofibrosarcoma Protuberans
}

National Cancer Institute

\section{Source}

National Cancer Institute. Dedifferentiated Dermatofibrosarcoma Protuberans. NCI

Thesaurus. Code C38107.

A morphologic variant of dermatofibrosarcoma protuberans characterized by the presence of poorly differentiated sarcomatous components. 